\title{
IDENTIFICATION OF GALAXY CLUSTER SUBSTRUCTURES WITH THE CAUSTIC METHOD
}

\author{
Heng Yu ${ }_{1}^{1,2,3}$, Ana Laura Serra ${ }^{4}$, Antonaldo Diaferio ${ }^{1,3}$, and Marco Baldi ${ }^{5,6,7}$ \\ ${ }^{1}$ Dipartimento di Fisica, Università di Torino, Via P. Giuria 1, I-10125 Torino, Italy \\ 2 Department of Astronomy, Beijing Normal University, Beijing 100875, China \\ ${ }^{3}$ Istituto Nazionale di Fisica Nucleare (INFN), Sezione di Torino, Via P. Giuria 1, I-10125 Torino, Italy \\ ${ }^{4}$ Dipartimento di Fisica, Università di Milano, Via Celoria 16, I-20133 Milano, Italy \\ ${ }^{5}$ Dipartimento di Fisica e Astronomia, Alma Mater Studiorum Università di Bologna, viale Berti Pichat, 6/2, I-40127 Bologna, Italy \\ ${ }^{6}$ INAF-Osservatorio Astronomico di Bologna, via Ranzani 1, I-40127, Bologna, Italy \\ INFN-Sezione di Bologna, viale Berti Pichat 6/2, I-40127, Bologna, Italy \\ Received 2015 March 2; accepted 2015 July 24; published 2015 August 26
}

\begin{abstract}
We investigate the power of the caustic technique for identifying substructures of galaxy clusters from optical redshift data alone. The caustic technique is designed to estimate the mass profile of galaxy clusters to radii well beyond the virial radius, where dynamical equilibrium does not hold. Two by-products of this technique are the identification of the cluster members and the identification of the cluster substructures. We test the caustic technique as a substructure detector on two samples of 150 mock redshift surveys of clusters; the clusters are extracted from a large cosmological $N$-body simulation of a $\Lambda$ CDM model and have masses of $M_{200} \sim 10^{14} h^{-1} M_{\odot}$ and $M_{200} \sim 10^{15} h^{-1} M_{\odot}$ in the two samples. We limit our analysis to substructures identified in the simulation with masses larger than $10^{13} h^{-1} M_{\odot}$. With mock redshift surveys with 200 galaxies within $3 R_{200}$, (1) the caustic technique recovers $\sim 30 \%-50 \%$ of the real substructures, and (2) $\sim 15 \%-20 \%$ of the substructures identified by the caustic technique correspond to real substructures of the central cluster, the remaining fraction being low-mass substructures, groups or substructures of clusters in the surrounding region, or chance alignments of unrelated galaxies. These encouraging results show that the caustic technique is a promising approach for investigating the complex dynamics of galaxy clusters.
\end{abstract}

Key words: galaxies: clusters: general - large-scale structure of universe - methods: numerical - methods: statistical

\section{INTRODUCTION}

Galaxy clusters, as the high-mass tail of the hierarchical structure, connect the large-scale structure to galaxies, and are thus relevant for constraining models of galaxy evolution, structure formation, and cosmology. Due to the large scale and long relaxing time of clusters, the presence of substructures is quite common, indicating that a number of clusters are likely to be out of equilibrium. Substructures can substantially affect the estimate of the cluster velocity dispersion and mass (Girardi et al. 1996; Pinkney et al. 1996), can provide insights into the formation process of the cluster, and unveil the existence of dark matter (Markevitch et al. 2004; Clowe et al. 2006). The presence of substructures appears to be a fundamental ingredient of the galaxy-environment connection and for shaping the morphology-density relation (e.g., Fasano et al. 2015; Girardi et al. 2015). The mass fraction in substructures can also probe structure formation and the expansion rate of the universe (Richstone et al. 1992; Kauffmann \& White 1993; Mohr et al. 1995; Thomas et al. 1998).

Attempts to identify and investigate cluster substructures have been numerous since their first discovery in the optical band (Shane \& Wirtanen 1954). In images of X-ray surface brightness, substructures are relatively obvious, especially with data coming from recent X-ray missions, including ROSAT (Kolokotronis et al. 2001; Schuecker et al. 2001), Chandra (Jeltema et al. 2005; Andrade-Santos et al. 2012; Parekh et al. 2014), and XMM-Newton (Zhang et al. 2009).

However, because the X-ray surface brightness decreases rapidly with increasing radius, these observations can only trace substructures in the central region of clusters. Substructures can also appear in microwave observations because free electrons in the hot X-ray gas originate from the SunyaevZeldovich effect (Komatsu et al. 2001; Korngut et al. 2011). The location of radio halos in clusters also tends to coincide with the substructures observed in X-ray images and temperature maps (see Feretti et al. 2012 for a recent review).

The existence of substructures in the dark matter halos of clusters can also be revealed by the anomalous images of strong gravitational lensing systems (Kneib et al. 1996; Mao \& Schneider 1998; Mao et al. 2004), or by peculiar features of the halo density profiles of weak lensing systems (Hoekstra et al. 2000; Clowe et al. 2006; Okabe et al. 2010; Pastor Mira et al. 2011; Oguri et al. 2013; McCleary et al. 2015; Shirasaki 2015), although the contamination by chance alignments of unrelated massive systems along the line of sight can be severe (Hoekstra 2003; Hoekstra et al. 2011; Geller et al. 2013).

The detection of substructures from optical data, based on the galaxy celestial coordinates and redshifts, is still a common approach for studying substructures. The methods can either use galaxy positions alone, redshifts alone, or both.

The methods that use galaxy positions alone include the smoothed density-contour maps (Geller \& Beers 1982), symmetry test, angular separations test, density contrast test (West et al. 1988), average two-point correlation function (Salvador-Solé et al. 1993), and two-dimensional (2D) wavelet transforms (Slezak et al. 1990; Escalera \& MacGillivray 1995; Flin \& Krywult 2006). They usually suffer from the contamination of background and foreground galaxies, but are clearly very useful when spectroscopic observations are missing or incomplete.

The methods that only use the galaxies' redshifts usually assume that the distribution of the velocities of the member 
galaxies is Gaussian. Based on this assumption, indicators like kurtosis, skewness (West \& Bothun 1990; Solanes et al. 1999), and the asymmetry and tail indices (Bird \& Beers 1993) are designed to quantify the subclustering in the one-dimensional (1D) redshift distribution. The 1D Kaye's mixture model (KMM) algorithm belongs to the set of Gaussian mixture model (GMM) methods: it assesses the presence of substructures by estimating the number of optimal partitions of Gaussian distributions (Ashman et al. 1994; Kriessler \& Beers 1997). The DEDICA method is based on an adaptive kernel and identifies specific velocity components (Pisani 1993); when the chosen kernel is Gaussian, DEDICA reduces to one of the GMM methods.

Among the methods using both the galaxy positions and redshifts, the Dressler \& Shectman (DS) method (Dressler \& Shectman 1988; Solanes et al. 1999; Knebe \& Müller 2000; Aguerri \& Sánchez-Janssen 2010; Dressler et al. 2013) is certainly the most widely used. Other methods include the three-dimensional (3D) KMM algorithm (Bird 1994; Colless \& Dunn 1996; Barmby \& Huchra 1998), which, in input, requires the number of the substructures and an initial guess of their positions; the 3D wavelet transforms (Escalera \& Mazure 1992; Gambera et al. 1997; Girardi et al. 1997; Pagliaro et al. 1999); the 3D version of DEDICA (Pisani 1996; Ramella et al. 2007); and the hierarchical tree algorithm (Serna \& Gerbal 1996; Adami et al. 2005).

All of these methods mainly focus on substructure detection. However, an unambiguous association of galaxies to individual substructures, which enables the derivation of the substructure properties, like size, velocity dispersion, and mass, is not yet available. Here we explore the possibility that the caustic technique can contribute a step forward in this direction.

The caustic technique (Diaferio \& Geller 1997; Diaferio 1999, 2009) estimates the escape velocity of cluster galaxies from the cluster center to a few times the virial radius. The technique name derives from the two curves in the redshift diagram where the galaxy number density is expected to be infinite in the spherical collapse model (Regös \& Geller 1989). With this technique, we can estimate the mass and gravitational potential profiles of galaxy clusters to radii that extend to the cluster infall region (see reviews in Diaferio 2009 and Serra et al. 2011). For the mass estimation, the caustic technique only assumes spherical symmetry and does not require the system to be in dynamical equilibrium. It can be used both in the central and in the outer regions of clusters, where other techniques cannot be applied.

The first step of the caustic technique procedure is to arrange the galaxies in a binary tree according to a projected pairwise galaxy binding energy. This step is similar to the procedure described in Serna \& Gerbal (1996). However, the caustic technique goes further and identifies a threshold that cuts the tree and identifies the cluster members. Serra \& Diaferio (2013) showed that this approach, combined with the location of the caustics in the cluster redshift diagram, returns a list of cluster members within $R_{200}$ that is $96 \%$ complete and only $2 \%$ of the members are actually interlopers. Within the larger radius $3 R_{200}$, where no other method is available, the completeness is $95 \%$ and the contamination is $8 \%$.

The same principle used to cut the binary tree and identify the cluster members provides a second threshold that gives a list of cluster substructures. So far, no systematic analysis of the properties of these substructures has been performed. Here, we use $N$-body simulations to investigate the power of the caustic technique to identify cluster substructures.

In Section 2, we describe the cosmological $N$-body simulation and the mock cluster redshift surveys we use to test the caustic technique as a substructure detector. We briefly review the caustic technique in Section 3. We present our results in Section 4, and we discuss them in Section 5.

\section{THE SIMULATED CLUSTER SAMPLES}

We use the Coupled Dark Energy Cosmological Simulations (Baldi 2012). This is the largest set to date of $N$-body simulations that model the interaction between the dark energy scalar field and the Cold Dark Matter (CDM) fluid. Here, however, we only consider the simulation of the standard $\Lambda C D M$ model with fiducial WMAP7 parameters. The simulated volume is a comoving cube of $1 h^{-1} \mathrm{Gpc}$ on a side ( $h=H_{0} / 100 \mathrm{~km} \mathrm{~s}^{-1} \mathrm{Mpc}^{-1}$ is the dimensionless Hubble constant), containing $1024^{3} \mathrm{CDM}$ particles with a mass of $5.84 \times 10^{10} h^{-1} M_{\odot}$ and the same number of baryonic particles with a mass of $1.17 \times 10^{10} h^{-1} M_{\odot}$. We only consider the dark matter particles: we assume that in the real universe galaxies are unbiased tracers of the velocity field of the dark matter particles. In fact, both $N$-body simulations (e.g., Diaferio et al. 2001; Diemand et al. 2004; Gill et al. 2004, 2005) and observations (e.g., Rines et al. 2008) indicate that any velocity bias between galaxies and dark matter is smaller than $10 \%$.

Halos are identified with the Friends-of-Friends (FoF) algorithm (Huchra \& Geller 1982; Davis et al. 1985), which links particles with distances less than the linking length $l_{\mathrm{FoF}}$ to form a group. We adopt the standard linking length $l_{\mathrm{FoF}}=0.2 l_{\text {mean }}$, with $l_{\text {mean }}$ as the mean interparticle separation, corresponding to the overdensity at viralization $\rho / \rho_{\mathrm{b}}=185$ (Audit et al. 1998), with $\rho_{\mathrm{b}}$ as the mean background density. In this procedure, the FoF halos are identified by using the CDM particles as primary tracers and then linking baryonic particles to the group of their closest CDM neighbor. The characteristic radius of the FoF halos, $R_{200}$, is the radius within which the average density (including both CDM and baryonic particles) is 200 times the critical density. The mass within $R_{200}$ is $M_{200}$.

We consider two samples of $50 \mathrm{FoF}$ halos, each at redshift $z=0$ : a massive sample (M15 hereafter), with $M_{200}$ ranging from $0.86 \times 10^{15} h^{-1} M_{\odot}$ to $3.4 \times 10^{15} h^{-1} M_{\odot}$, and median $1.1 \times 10^{15} h^{-1} M_{\odot}$; and a less massive sample (M14 hereafter), with masses ranging from $0.95 \times 10^{14} h^{-1} M_{\odot}$ to $1.1 \times 10^{14} h^{-1} M_{\odot}$, and median $1.0 \times 10^{14} h^{-1} M_{\odot}$.

For each cluster, we compile three mock galaxy redshift catalogs. Each cluster is located at the center of the volume using the periodic boundary conditions of the simulation box. We assign the celestial coordinates $(\alpha, \delta)=\left(6^{\mathrm{h}}, 0^{\circ}\right)$ and a redshift distance $c z=36,000 \mathrm{~km} \mathrm{~s}^{-1}$ to the cluster center. Around the cluster, we consider a rectangular prism enclosing the volume, corresponding to a solid angle that at the cluster distance covers a square area $12 h^{-1} \mathrm{Mpc}$ wide. The volume is centered at the cluster and is $140 h^{-1} \mathrm{Mpc}$ deep. The resulting field of view $(\mathrm{FOV})$ is $1: 6 \times 1: 6$. For each cluster, we apply this procedure to three orthogonal directions. Since the clusters are generally not spherically symmetric, for our statistical purposes we can consider these three mock catalogs as independent clusters. We thus obtain 150 mock redshift catalogs for each of the samples (M15 and M14).

The observational volumes we extract from the simulation typically contain $\sim 8 \times 10^{4}$ particles for the M15 sample and 
Table 1

The Number of Particles $N$ in the FOV

\begin{tabular}{lcrrrrrr}
\hline \hline & \multicolumn{3}{c}{ M15 (1500 clusters) } & & \multicolumn{3}{c}{ M14 (1500 clusters) } \\
\cline { 2 - 3 } \cline { 7 - 8 }$N_{3 R}$ & $10 \%$ & $50 \%$ & $90 \%$ & & $10 \%$ & $50 \%$ & $90 \%$ \\
\hline 100 & 185 & 241 & 325 & 672 & 996 & 1490 \\
200 & 369 & 481 & 644 & & 1356 & 2014 & 2905 \\
300 & 553 & 718 & 969 & & 2034 & 2988 & 4385 \\
400 & 739 & 962 & 1283 & & 2704 & 4016 & 5829 \\
500 & 923 & 1195 & 1603 & 3394 & 5034 & 7258 \\
\hline
\end{tabular}

$\sim 5 \times 10^{4}$ particles for the M14 sample. Realistic numbers of observable galaxies in these volumes are clearly much smaller. Therefore, we randomly sample the dark matter particles until we obtain a given number of particles $N_{3 R}$ within $3 R_{200}$. To explore the effect of galaxy sampling, we build catalogs with $N_{3 R}=(100,200,300,400$, and 500). Additionally, we only retain particles within $\pm 4000 \mathrm{~km} \mathrm{~s}^{-1}$ from the cluster center. These mock galaxy redshift surveys of cluster regions are roughly comparable to recent large surveys of clusters and their surroundings, such as CIRS (Rines \& Diaferio 2006) and HeCS (Rines et al. 2013).

A different strategy to build mock redshift surveys could be to keep the number of particles in the FOV for both the M14 and M15 samples fixed. However, this procedure returns mock surveys where the substructures of the M14 clusters are poorly sampled or, more often, not sampled at all, because as mentioned above the observational volumes of the M14 sample are on average $60 \%\left(5 \times 10^{4} / 8 \times 10^{4}\right)$ less populated than the M15 sample volumes. On the contrary, keeping $N_{3 R}$ fixed guarantees that we properly sample the substructures and guarantees that it is closer to the observational procedure of surveys dedicated to the study of the dynamics of clusters; these surveys tend to sample the volume more densely around the cluster, both on the sky and in redshift space. The very different final numbers of particles in the FOV of the M14 and M15 samples (Table 1) reflects a real effect: identifying substructures in less massive clusters requires denser surveys because in these clusters the probability of measuring the redshift of a galaxy that does not belong to the cluster is larger.

For a given $N_{3 R}$, the total numbers of particles $N$ in the FOV of a mock cluster depend on the cluster and its surrounding region. To investigate the effects of the fluctuations caused by random sampling, we repeat the procedure 10 times. Table 1 lists the medians and percentile ranges of the number of particles $N$ as a function of $N_{3 R}$ : for example, $80 \%$ of the mock catalogs of the M15 sample with $N_{3 R}=100$ have $N$ in the range of $185-325$. For our statistical purposes, these 10 random realizations of each individual line-of-sight projection of a given cluster with a fixed $N_{3 R}$ cannot be considered independent. Hereafter, we will only use these 10 realizations to quantify the fluctuations of the random sampling on the cluster samples M15 and M14. Finally, in the M14 FOV's $N$ is four to five times larger than in the M15 samples: because the M15 clusters are an order of magnitude more massive than the M14 clusters, whereas the surrounding cosmic volumes are comparable, in the M15 samples the random sampling reaches $N_{3 R}$ more rapidly and the number of particles sampled in the surrounding region is proportionally smaller.

To identify the substructures of the clusters in the simulations, we use the code SUBFIND (Springel et al. 2001), whose algorithm is based on the overdensity and the gravitational binding energy of the particles. More specifically, for each FoF halo detected by the FoF algorithm, SUBFIND identifies candidate substructures by sorting the particles of the FoF halo according to their local density and isolating local density maxima. This procedure provides substructures whose boundaries are determined by the first saddle point identified in the local density field around each density maximum. From each substructure, we finally remove all particles with positive total energy (see Baldi 2012 for further details).

The mass of a substructure is always its total mass, namely the sum of the mass of the particles (both CDM and baryons) that are gravitationally bound to that substructure as identified by SUBFIND. The first row of Table 2 lists the total number of substructures with masses larger than $10^{13} h^{-1} M_{\odot}$ in our two samples M15 and M14. The $10^{13} h^{-1} M_{\odot}$ mass threshold is not arbitrary, but is a minimum substructure mass set by the number of luminous galaxies that can be detected in current typical surveys. In fact, a $10^{13} h^{-1} M_{\odot}$ substructure is expected to contain at most a handful of galaxies brighter than $L_{*}$. Hereafter, we will call these substructures 3D substructures.

By randomly sampling the dark matter particles, the number of members of a 3D substructure in the mock catalog can be substantially reduced or even vanish. We only consider 3D substructures that have at least 10 particles appearing in the FOV.

Table 2 lists the total number of clusters $N_{\mathrm{cl}}$ with at least one $3 \mathrm{D}$ substructure appearing in the FOV, the total number of 3D substructures in all the FOVs $N_{\text {sub }}$, and the ratio between these detectable substructures and the total number of substructures $\left(N_{\text {sub }} / N_{\text {tot }}\right)$; the total number of substructures $N_{\text {tot }}$ is listed in the first row of Table 2. As expected, the number of 3D substructures appearing in the FOV increases with increasing $N_{3 R}$. We also list the standard deviations deriving from the ten random realizations. We see that the random sampling has a moderate impact. In the M15 samples, the number of clusters that do not show 3D substructures in the FOV is substantial: if we consider the total members of the cluster as the sum of the members of the 3D substructures and the members of the cluster core identified by SUBFIND, $(36 \pm 8) \%$ of the total members belong to the 3D substructures in the M14 samples, whereas this fraction is only $(11 \pm 6) \%$ in the M15 samples. In addition, as mentioned earlier, the M15 FOV's are four to five times less populated than the M14 fields. Therefore, random sampling makes 3D substructures in the M15 samples vanish more easily than in the M14 samples.

\section{THE CAUSTIC METHOD}

According to hierarchical clustering models, clusters of galaxies form by the aggregation of smaller systems. The local gravitational potential plays a crucial role in determining the galaxy velocities in addition to the radial infall expected in the spherical collapse model (Diaferio \& Geller 1997). On the redshift diagram of the line-of-sight velocity $v$ of the galaxies in the cluster rest frame versus their projected distance $r$ from the cluster center, the cluster members populate a trumpet-shaped region that is approximately symmetric around the $r$ axis (Kaiser 1987; Regös \& Geller 1989; van Haarlem \& van de Weygaert 1993). The caustics define the boundaries of this region, whose amplitude $\mathcal{A}(r)$ decreases with increasing $r$. $\mathcal{A}(r)$ provides the estimate of the escape velocity profile from 
Table 2

Number of Clusters with 3D Substructures in the FOV and Number of 3D Substructures

\begin{tabular}{|c|c|c|c|c|c|c|}
\hline \multirow[b]{2}{*}{$N_{3 R}$} & \multicolumn{3}{|c|}{ M15 } & \multicolumn{3}{|c|}{ M14 } \\
\hline & $N_{\mathrm{cl}}$ & $N_{\text {sub }}$ & Ratio (\%) & $N_{\mathrm{cl}}$ & $N_{\text {sub }}$ & Ratio (\%) \\
\hline$\ldots$ & 150 & $594^{\mathrm{a}}$ & $\ldots$ & 150 & $282^{\mathrm{a}}$ & $\ldots$ \\
\hline 300 & $61 \pm 4$ & $64 \pm 4$ & 10.8 & $150 \pm 0$ & $275 \pm 3$ & 97.5 \\
\hline 400 & $81 \pm 4$ & $94 \pm 5$ & 15.8 & $150 \pm 0$ & $282 \pm 1$ & 100 \\
\hline 500 & $99 \pm 3$ & $132 \pm 6$ & 22.2 & $150 \pm 0$ & $282 \pm 0$ & 100 \\
\hline
\end{tabular}

Note.

${ }^{\mathrm{a}} N_{\text {tot }}$, total number of $3 \mathrm{D}$ substructures with masses larger than $10^{13} h^{-1} M_{\odot}$.

the cluster and thus its mass profile (Diaferio \& Geller 1997; Diaferio 1999).

To measure $\mathcal{A}(r)$, the caustic technique builds a binary tree based on the projected galaxy pairwise energy, determines a threshold to cut the binary tree, identifies a set of candidate cluster members that in turn determines the cluster center and defines the redshift diagram. The caustic technique locates the caustics and thus $\mathcal{A}(r)$ from the galaxy number density on the redshift diagram. The steps that are relevant for the identification of the substructures we are interested in here are the construction of the binary tree and its threshold determination. For the sake of completeness, we list the details of these steps. Further details are provided in Diaferio (1999) and Serra et al. (2011).

To build the binary tree, we proceed as follows:

i. initially each galaxy is a group $g_{\alpha}$;

ii. the binding energy $E_{\alpha \beta}=\min \left\{E_{i j}\right\}$, where $E_{i j}$ is a projected binding energy between the galaxy $i \in g_{\alpha}$ and the galaxy $j \in g_{\beta}$, is associated to each group pair $g_{\alpha}, g_{\beta}$. The projected binding energy is estimated with the relation

$$
E_{i j}=-G \frac{m_{i} m_{j}}{R_{\mathrm{p}}}+\frac{1}{2} \frac{m_{i} m_{j}}{m_{i}+m_{j}} \Pi^{2},
$$

where $R_{\mathrm{p}}$ is the pair projected separation, $\Pi$ is the line-ofsight velocity difference and $m_{i}=m_{j}=10^{12} h^{-1} \mathrm{M}_{\odot}$ is the typical total mass of a luminous galaxy;

iii. the two groups with the smallest binding energy $E_{\alpha \beta}$ are replaced with a single group $g_{\gamma}$ and the total number of groups is decreased by one; and

iv. the procedure is repeated from step (ii) until only one group is left.

At this stage all the galaxies are arranged in a binary tree; an example is shown in Figure 1. This dendrogram is derived from a mock catalog of the M14 sample with $N_{3 R}=100$ and $N=606$. To identify the members of the cluster and its substructures, we need to cut the tree at some level. Toward this aim, we identify the main branch from the root to the leaves by tracing the node that contains the largest number of galaxies at each bifurcation. The leaves that hang from each node $x$ of the main branch provide a velocity dispersion $\sigma_{\text {los }}^{x}$. When walking along the main branch from the root to the leaves, $\sigma_{\text {los }}^{x}$ rapidly decreases due to the progressive loss of galaxies that are most likely not associated with the cluster (Figure 2); then $\sigma_{\text {los }}^{x}$ reaches a " $\sigma$ plateau" at some node $x_{1}$.
Most of the galaxies hanging from this node are members of the cluster: in fact, the system is nearly isothermal and moving along the main branch toward the leaves removes the less bound galaxies that in general do not substantially affect the value of $\sigma_{\text {los }}^{x}$. When we get close to the leaves along the main branch, the remaining galaxies have a binding energy that is very small and causes $\sigma_{\text {los }}^{x}$ to drop again. This second rapid drop identifies the node $x_{2}$ that sets the limit of the $\sigma$ plateau.

To identify the $\sigma$ plateau and its boundaries $x_{1}$ and $x_{2}$, Serra et al. (2011) designed an algorithm based on the distribution of the velocity dispersions of the nodes, as detailed below.

i. Derive the probability density distribution of the velocity dispersion $\sigma_{\text {los }}^{x}$ of the leaves hanging from each node; an example is shown in the right panel of Figure 2. The mode of this distribution corresponds to the value $\sigma_{\mathrm{pl}}$ of the $\sigma$ plateau.

ii. To identify the nodes belonging to the $\sigma$ plateau, (1) we remove the tails beyond $\pm 0.3 \sigma_{\mathrm{pl}}$ of the $\sigma_{\text {los }}^{x}$ distribution, and (2) the $80 \%$ of the remaining nodes closest to $\sigma_{p l}$ are retained as the $N_{\delta}$ nodes defining the $\sigma$ plateau.

iii. We choose $x_{1}$ among the first (i.e., closest to the root) five nodes of the set of the $N_{\delta}$ nodes, as the node whose $\sigma_{\text {los }}^{x}$ has the smallest discrepancy from $\sigma_{\mathrm{pl}}$; similarly we choose $x_{2}$ among the last five nodes (i.e., furthest away from the root).

The first node, $x_{1}$, closest to the root, is the appropriate level for the identification of the cluster. The threshold set by node $x_{1}$ separates the binary tree branches into different groups. The group containing the main branch is the main group and its galaxies are the candidate members of the cluster. The completeness and purity of these candidate members have been investigated by Serra \& Diaferio (2013). We consider all of the other groups separated by the threshold $x_{1}$ dynamically distinct from the cluster, or the main group, and we disregard them hereafter.

The second node, $x_{2}$, farthest away from the root, identifies the substructure candidates. We define all the substructures, whose members belong to the main group, as the 2D substructures of the cluster. We only consider 2D substructures with at least 10 particles. We disregard all of the systems separated by the threshold set by node $x_{2}$ whose members do not belong to the main group. As an example, Figure 3 shows the distribution on the sky of the identified groups and the 2D substructures according to the dendrogram and $\sigma$ plateau of Figures 1 and 2. 


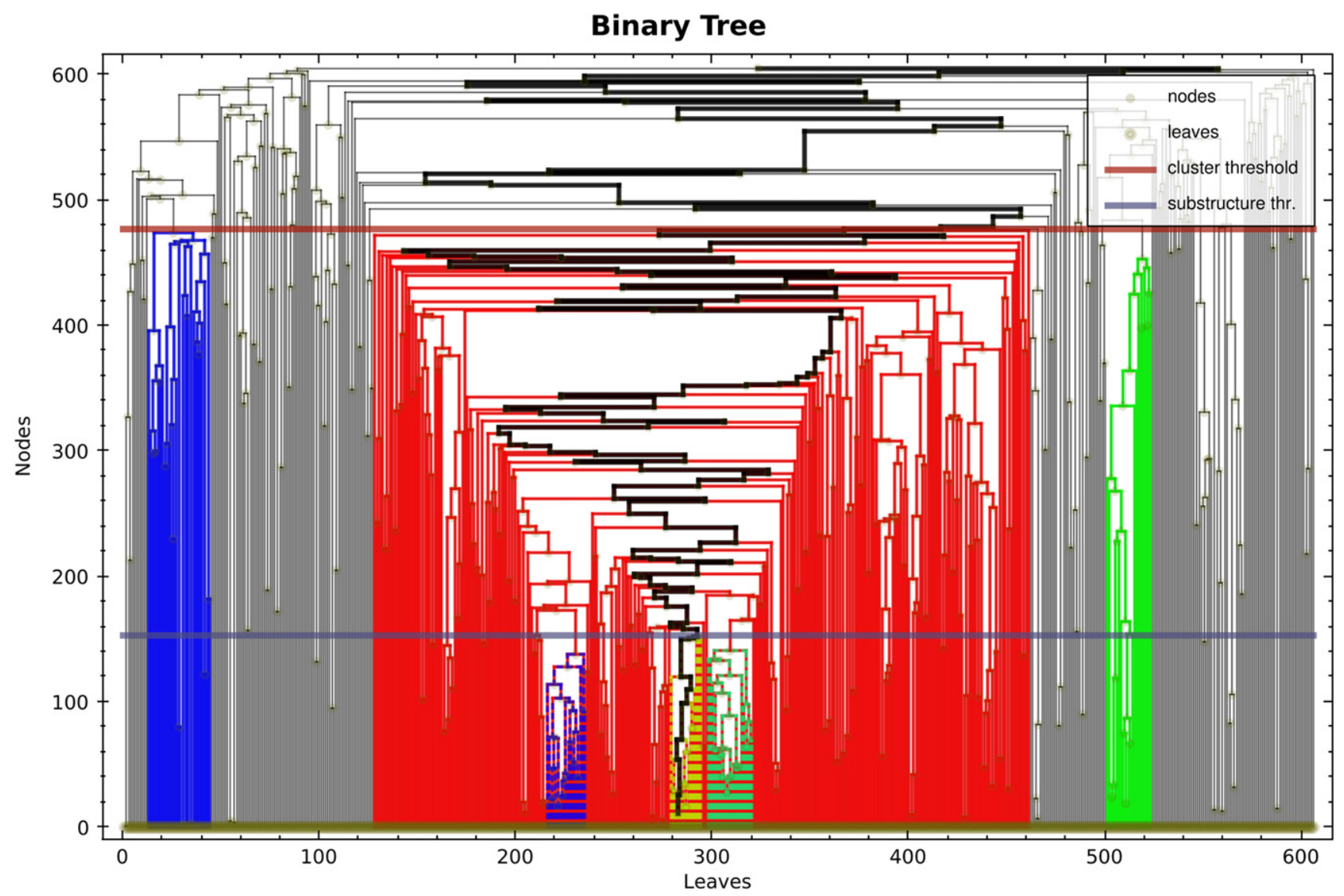

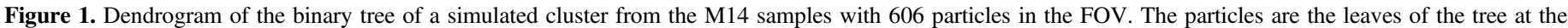

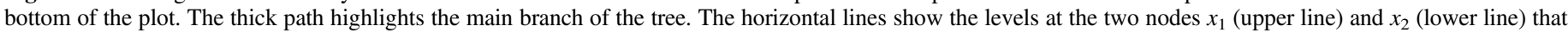

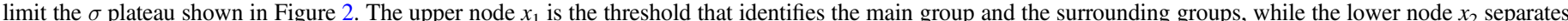

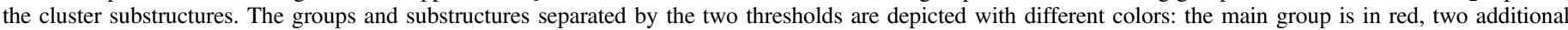

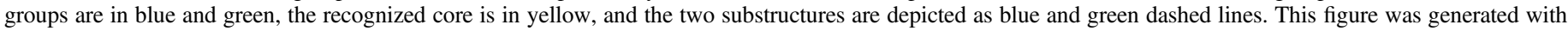
the software CausticApp (Serra and Diaferio, personalpages.to.infn.it/ serra/causticapp.html).

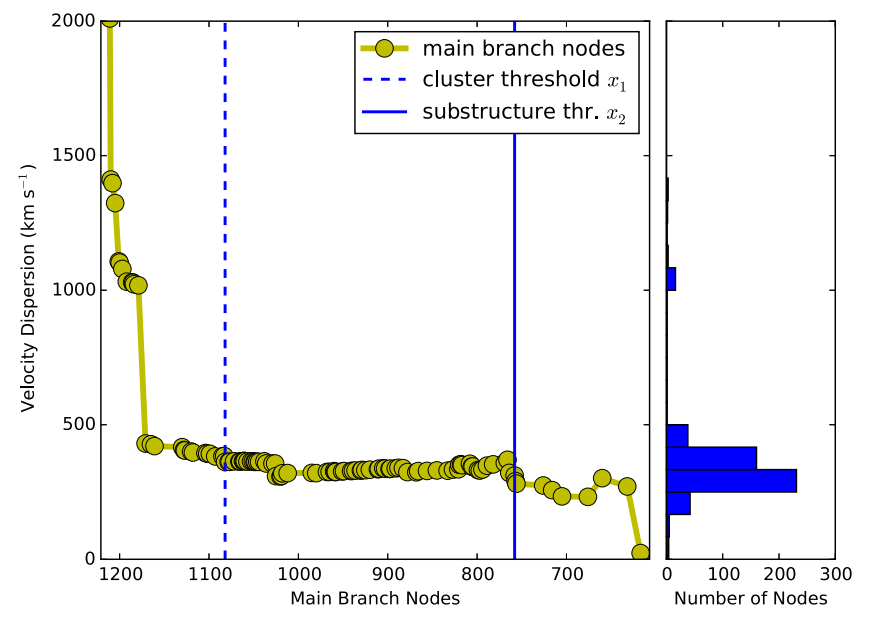

Figure 2. Velocity dispersion of the leaves of each node along the main branch of the binary tree shown in Figure 1. The vertical dashed and solid lines show the nodes $x_{1}$ and $x_{2}$, respectively. The curve between $x_{1}$ and $x_{2}$ is the $\sigma$ plateau, whose position is indicated by the peak of the histogram of node numbers shown in the right panel.

\section{ANALYSIS AND RESULTS}

\subsection{Cluster Identification}

Because of the random sampling of particles, some substructures might have less than 10 particles in the FOV.
According to our limit, these substructures are not taken into account. If all the 3D substructures vanish from the FOV, the cluster has no substructures left. The height of the bars of each $N_{3 R}$ bin in Figure 4 shows the total fraction of clusters that have $3 \mathrm{D}$ substructures in the FOV. These fractions correspond to the number of clusters listed in Table 2. As noted in the previous section, almost all of the clusters in the M14 samples show 3D substructures, whereas many clusters of the M15 samples have had all of their 3D substructures disappear.

When constructing the binary tree from the data set of a cluster redshift survey, the main group of the binary tree might identify a system different from the cluster we are interested in because this cluster might not be the richest system in the FOV. With real data sets, where we usually analyze the clusters individually, we can easily correct for this situation by reducing the area of the FOV or by imposing the desired cluster center. Here, where we analyze large samples of mock clusters automatically and blindly, we simply remove these cases. To check whether the main group identifies the correct cluster, we compare the 2D members, namely the members of the main group, with the 3D members of the cluster core identified by SUBFIND. We say that a cluster is correctly identified if at least $60 \%$ of its 3D members are in the list of the $2 \mathrm{D}$ members. In Figure 4 the sum of the blue and cyan sectors of the bars shows the fraction of correctly identified clusters; the red sectors of the bars show the fraction of misidentified clusters. 

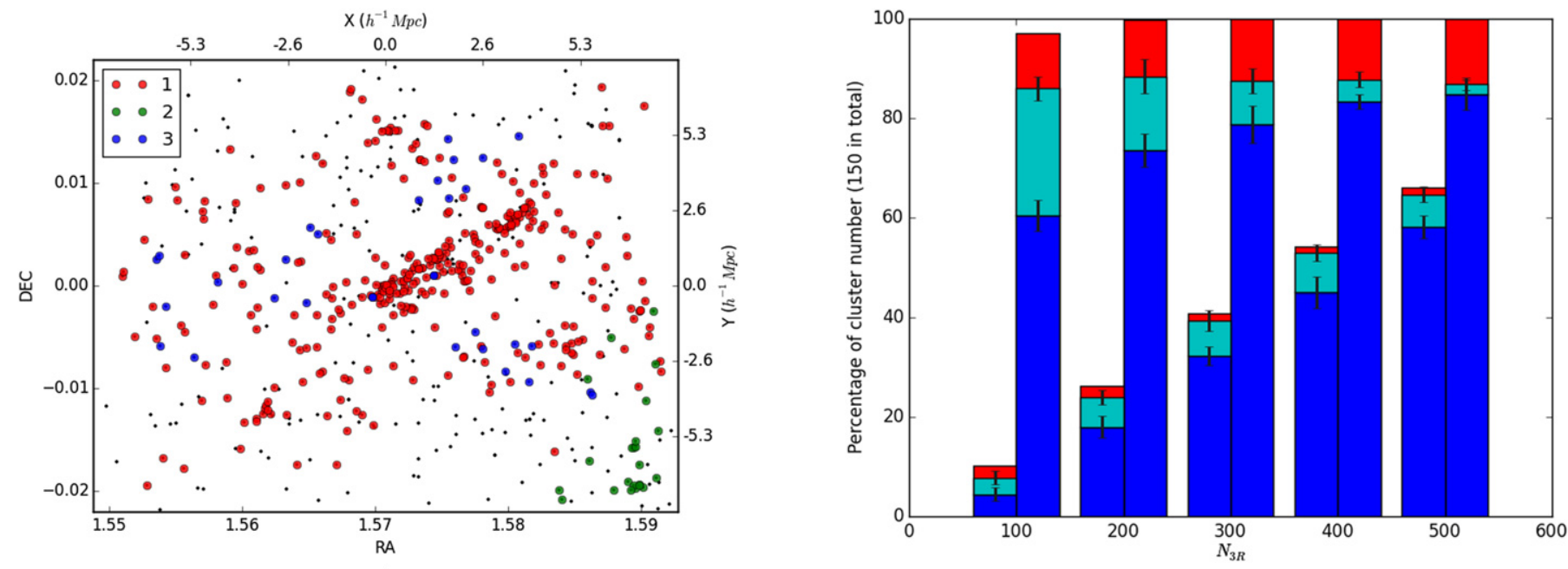

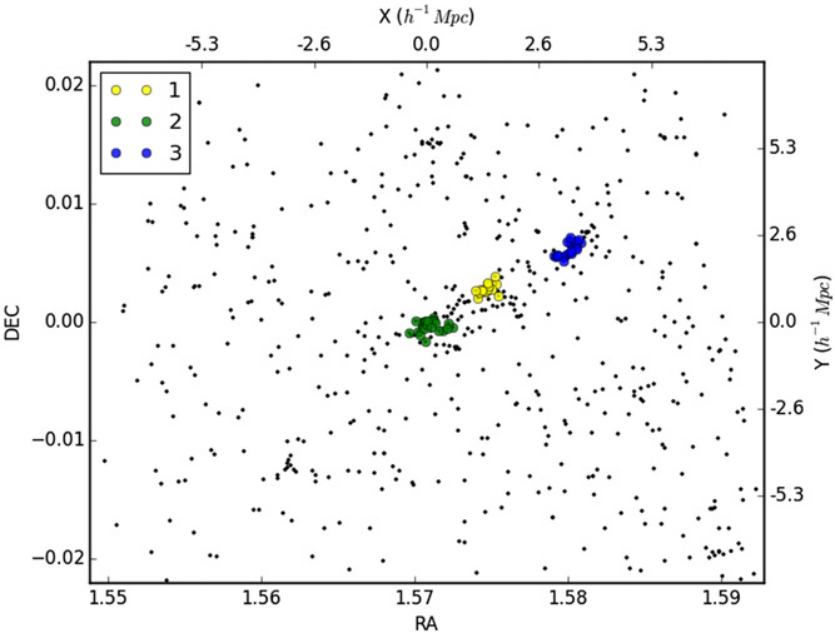

Figure 3. Sky diagrams of the groups (upper panel) and 2D substructures (lower panel) of the cluster whose dendrogram and $\sigma$ plateau are shown in Figures 1 and 2. The projected celestial coordinates are in radiants; comoving coordinates in the $\mathrm{N}$-body simulation are also shown. Particles with the same color belong to the same structure identified by the binary tree: clusters and groups in the upper panel, and substructures in the lower panel. The color code is the same as in Figure 1.

The fraction of correctly identified clusters is further split into the fraction of clusters with $2 \mathrm{D}$ substructures, shown by the blue sectors, and the fraction of clusters with no 2D substructures, shown by the cyan sectors. The lack of 2D substructures is usually caused by a relatively low second threshold $x_{2}$ that does not leave a sufficiently large number of particles for the substructure identification.

In passing, we note that we confirm the results of Serra \& Diaferio (2013), who find that on average, in clusters identified by the $\sigma$ plateau, $13 \%$ of the members within $3 R_{200}$ are actually interlopers (see their Table 1, 7th column, 9th row). Therefore, increasing our $60 \%$ threshold only slightly decreases the fraction of correctly identified clusters, for example, only by $1 \%$ if we increase the threshold to $80 \%$. Substructures are more poorly populated and proportionally more difficult to detect than clusters. As we see below, for substructures, a $60 \%$ threshold turns out to be a reasonable compromise between the completeness and the success rate: we thus also use the $60 \%$ threshold for the clusters to adopt a single criterion for both structures.
Figure 4. Fraction of clusters with $3 \mathrm{D}$ substructures in the FOV. Around each $N_{3 R}$, the left (right) bar is for the M15 (M14) sample. The blue sectors show the fraction of correctly identified clusters with $2 \mathrm{D}$ substructures. The cyan sectors show the fraction of correctly identified clusters without $2 \mathrm{D}$ substructures. The red sectors show the remaining fraction of clusters that are not on the main branch of the binary tree. The error bars show the standard deviations of these fractions deriving from the ten random sampling realizations.

\subsection{D Versus 3D Substructures: The Success Rate}

To quantify whether the 2D substructures correspond to the 3D substructures, we make a one-to-one comparison between the members of the 2D substructures identified by the binary tree and the members of the $3 \mathrm{D}$ substructures identified by SUBFIND. A single 2D substructure may contain members belonging to different 3D substructures or none. We find that in all of the M15 and M14 samples combined, $49 \%$ of the 2D substructures contain at least one member of a 3D substructure. For each of these $2 \mathrm{D}$ substructures, we define $f_{3 \mathrm{D}}$ as the largest fraction of its total number of members that are also members of a single 3D substructure. Figure 5 shows the distribution of $f_{3 \mathrm{D}}: 51 \%$ of the $2 \mathrm{D}$ substructures have an $f_{3 \mathrm{D}}$ larger than 0.8 . We adopt $f_{3 \mathrm{D}}=0.6$, a value smaller than the median $f_{3 \mathrm{D}}=0.8$, as the threshold to consider a 3D substructure successfully identified by a 2D substructure. Adopting a smaller threshold increases the success rate at the expense of increasing the discrepancy between the properties of the 2D and $3 \mathrm{D}$ substructures. A larger threshold makes the identification more solid, but substantially drops the success rate.

It can happen that different 2D substructures contain members of the same 3D substructure. This event occurs for $12 \%$ of the $2 \mathrm{D}$ substructures of the M14 samples and for $1.2 \%$ of the 2D substructures of the M15 samples. In these cases, we take the 2D substructure containing the largest number of the $3 \mathrm{D}$ substructure members as the match to the 3D substructure.

Figure 6 shows a random example of the substructure identification. The cluster has only one 3D substructure, whose center is indicated by the yellow star. The caustic method returns seven $2 \mathrm{D}$ substructures in addition to the cluster core, indicated by the yellow square, which is correctly matched. One of the 2D substructures correctly coincides with the 3D substructure. Out of the remaining six 2D substructures that do not correspond to any 3D substructure of the cluster identified with SUBFIND, two are close to the core and four are relatively distant from the cluster center. We consider these six 2D substructures to be false detections. 


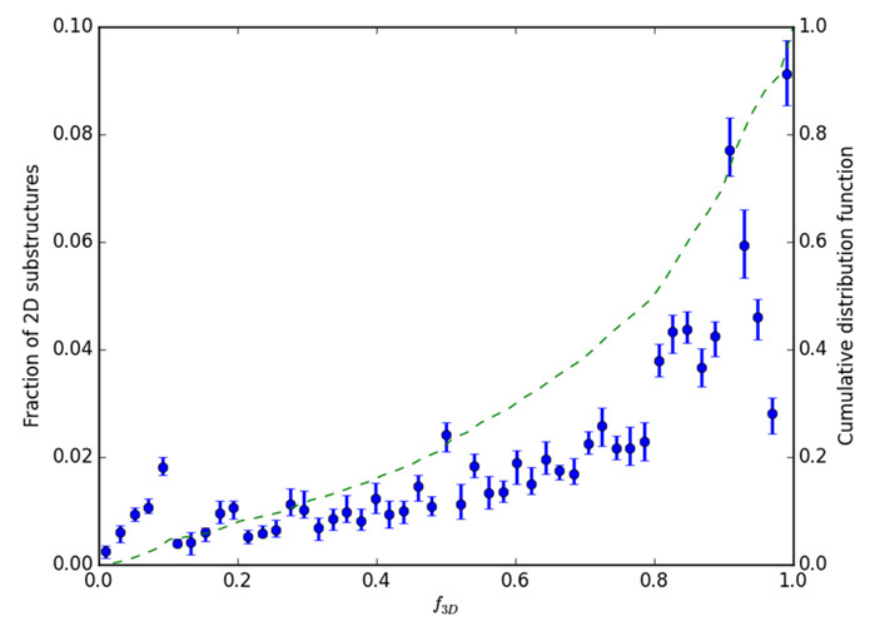

Figure 5. Distribution of $f_{3 \mathrm{D}}$, the largest fraction of the total number of members of a 2D substructure that are also members of a single 3D substructure. The error bars show the $10 \%$ and $90 \%$ percentile ranges from the ten random sampling realizations. The dashed line is the cumulative distribution function.

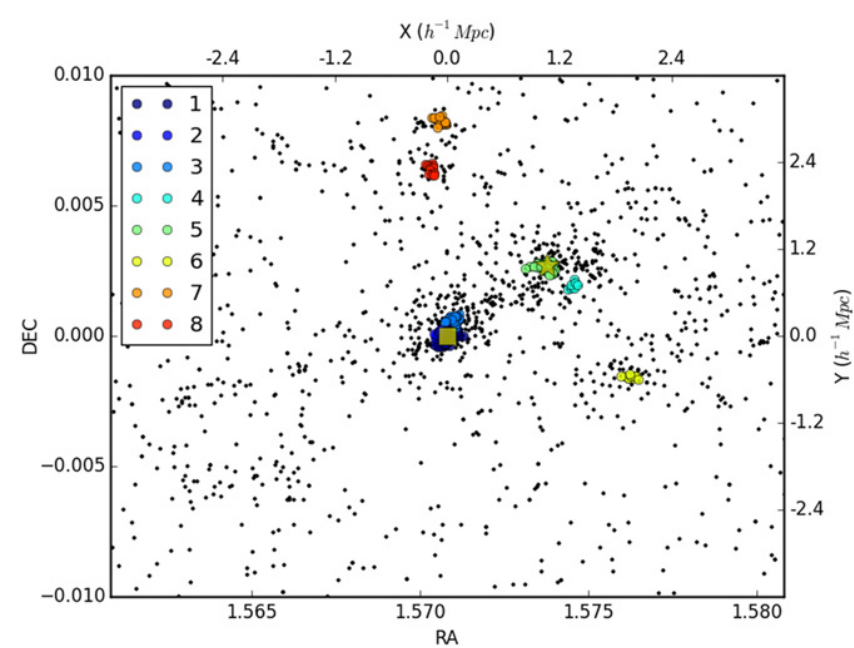

Figure 6. Example of the identification of substructures with the caustic technique. This cluster from the M14 sample contains 500 particles within $3 R_{200}$, with 4021 dark matter particles in the FOV (black dots). Here we only show a fourth of the original FOV area around the cluster center indicated by the square. The star shows the center of the only 3D substructure with a mass larger than $10^{13} h^{-1} M_{\odot}$. The colored circles show the members of the seven 2D substructures and of the cluster core identified with the caustic technique.

In fact, in our statistical analysis, we consider as false detections all the 2D substructures that do not correspond to the 3D substructures that SUBFIND associates with the analyzed cluster. However, our choice is rather restrictive. Figure 7 shows the 3D distribution of the system shown in Figure 6: out of the six 2D substructures, only one system is due to chance alignment; the remaining five 2D substructures are clearly bound systems. Two of them, close to the cluster core, are not in our list of 3D substructures because they have masses smaller than $10^{13} h^{-1} M_{\odot}$. The remaining three 2D substructures are groups surrounding the cluster center. As mentioned above, we do not include them in our list of 3D substructures in order to restrict our analysis to the 3D substructures of the analyzed cluster.

This random example shows that the rate of successful detections and the completeness we will show below are clearly

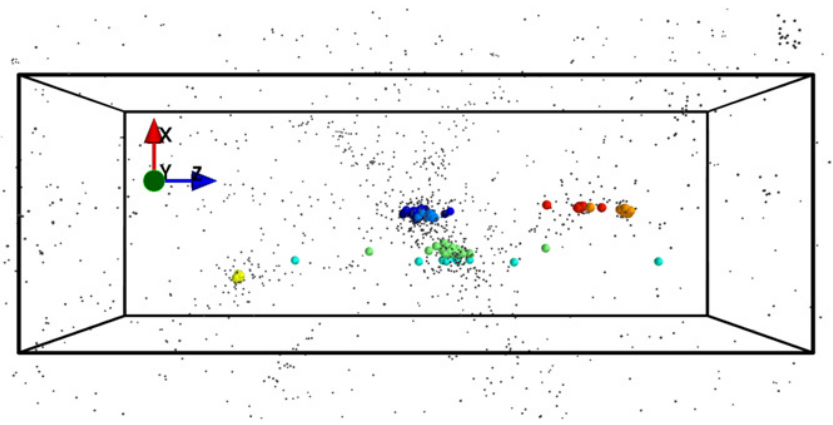

Figure 7. The 3D distribution of the system shown in Figure 6. To provide the scale and a 3D perspective, we overplot a box with dimensions $7 \times 7 \times 20 h^{-3} \mathrm{Mpc}^{3}$. The symbols and colors are as in Figure 6 .

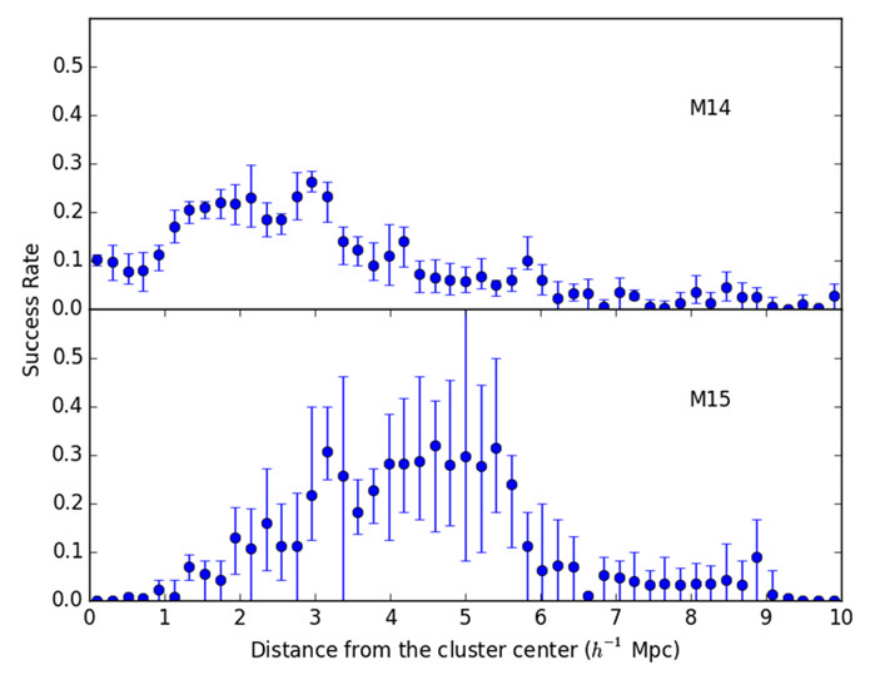

Figure 8. Success rate as a function of the projected distance of the $2 \mathrm{D}$ substructures from the cluster center. The error bars show the $10 \%$ and $90 \%$ percentile ranges from the ten random sampling realizations.

correct in the context of focusing on the massive substructures of individual clusters, but are likely to be lower limits to the performance of the identification of bound systems from redshift data with the caustic technique.

Figures 6 and 7 suggest that the 2D substructures at large projected distances from the cluster center are likely to be groups and substructures of surrounding clusters. We thus compute the success rate, namely the ratio between the number of 2D substructures that correspond to 3D substructures and the total number of 2D substructures, as a function of distance from the cluster center. Figure 8 shows this relation for the combined M14 and M15 samples. The success rate peaks at different radii in the two samples because the clusters have different sizes. When the radii are normalized to $R_{200}$, both peaks appear in the range $\sim 2-3 R_{200}$. We keep the length in this plot in proper units, because these units are more convenient with real data; in addition, the mass distribution of each cluster sample is very peaked and the distance normalization plays a little role within the same cluster sample. The success rate shows a broad peak between $\sim 1$ and $\sim 3 h^{-1}$ Mpc for the M14 samples and between $\sim 3$ and $\sim 5 h^{-1} \mathrm{Mpc}$ for the M15 samples, whereas it decreases at the center and in the outskirts of the clusters. The low rate at small distances is due to the cases where the cluster core is identified as a substructure rather than as the core itself. Again, we consider these cases to be false detections because we are interested in the 3D substructure identification, although these 


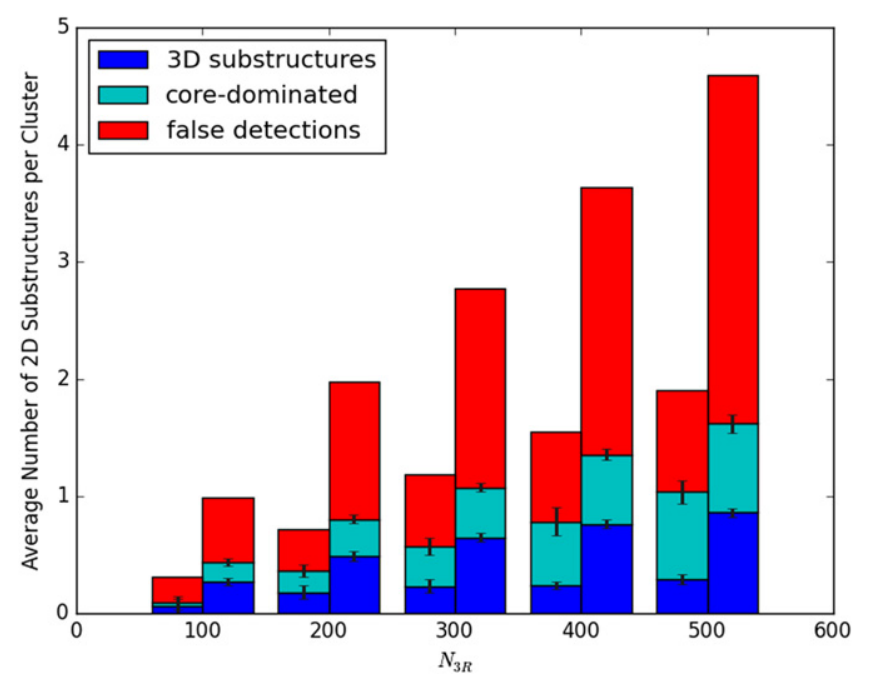

Figure 9. Average number of 2D substructures per cluster as a function of $N_{3 R}$ : The left (right) bars are for the M15 (M14) samples. The blue sectors show the 2D substructures that correspond to 3D substructures; the cyan sectors show the $2 \mathrm{D}$ substructures that are actually part of the cluster core; the red sectors show the false detections. The error bars show the $1 \sigma$ deviation from the 10 random sampling realizations.

identified 2D substructures actually are bound systems. In the cluster outskirts, the number of 3D substructures clearly decreases, unlike the number of 2D substructures. Therefore, we can introduce a distance criterion by removing all of the $2 \mathrm{D}$ substructures at distances outside a given range that, based on Figure 8 , we arbitrarily choose to be $[0.1,6] h^{-1} \mathrm{Mpc}$ : this criterion can remove most of the many false detections without missing promising 2D substructures.

Figure 9 shows the success rate as a function of $N_{3 R}$, with the distance criterion applied. The average number of $2 \mathrm{D}$ substructures increases with $N_{3 R}$ as expected, whereas the success rate decreases from $25 \%$ at $N_{3 R}=100$ to $15 \%$ at $N_{3 R}=500$ (these fractions can be read off in Figure 9 from the ratio between the length of the blue sector of each bar and the total length of the bar): in fact, a larger number of particles in the FOV increases the sampling of the 3D substructures, but at the same time it increases the probability of detecting 2D substructures by chance alignment. The cyan sectors of the bars show the fraction of 2D substructures whose members are 3D members of the cluster core rather than members of the 3D substructures. The error bars show that the random sampling fluctuations have a small impact.

We can look at our results from a different perspective. Figure 10 shows the distribution of the success rate. The $y$ - and $x$-axes show $N_{3 R}$ and the success rate, respectively. The gray scale shows the fraction of clusters on this plane. For example, the bottom row of the bottom panel shows that $63 \%$ of the clusters in the M15 sample with $N_{3 R}=100$ have no successful detection, whereas in $34 \%$ of the clusters all of the 2D substructures correspond to real 3D substructures.

This figure clearly shows that clusters are not uniformly distributed on this plane. In fact, the number of 2D substructures is discrete and may be small, with just one or two 2D substructures, especially when $N_{3 R}$ is small. Figure 10 also shows that in all of the M15 samples, about $60 \%$ of the clusters have 2D substructures that are all false. This result is due to the fact that there are fewer particles belonging to $3 \mathrm{D}$ substructures in the FOV of massive clusters (see Section 2).

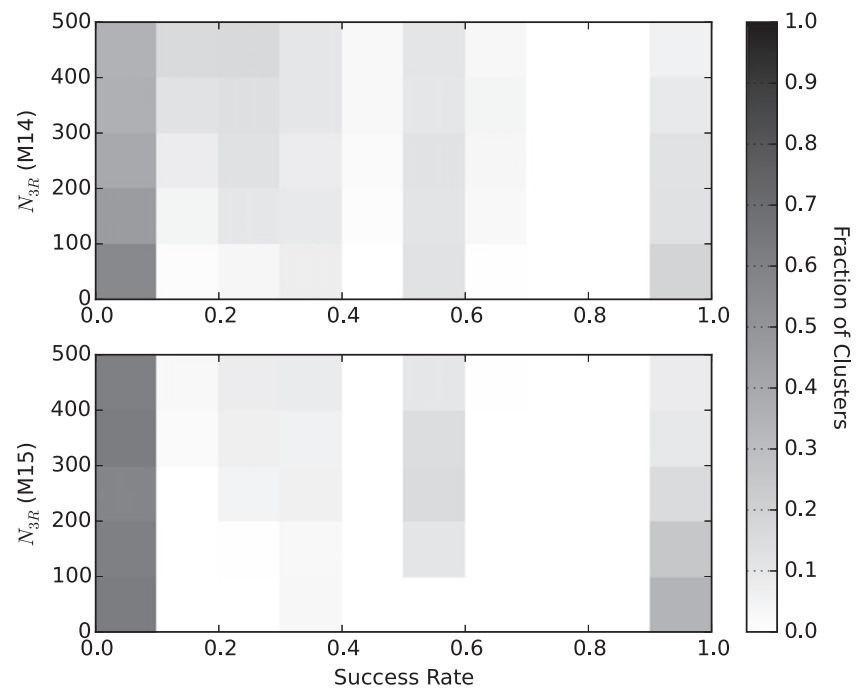

Figure 10. Distribution of the clusters in the plane $N_{3 R}$ vs. success rate. The gray scale shows the number of clusters normalized to the total number of clusters with $3 \mathrm{D}$ substructures in the FOV.

The lack of substructures makes the boundary of the $\sigma$ plateau quite ambiguous and consequently the identification of the second threshold $x_{2}$ becomes more problematic. On the contrary, the success rate in the M14 samples is more equally distributed on the plane and the fraction of false 2D substructures is proportionally smaller.

\subsection{Completeness}

We now estimate the completeness of our samples of substructures, namely the ratio between the number of correctly identified 3D substructures and the total number of 3D substructures. We note that the total number of 3D substructures only includes the $3 \mathrm{D}$ substructures more massive than $10^{13} h^{-1} M_{\odot}$ and with at least 10 particles in the FOV.

The completeness of the individual samples is shown in Figure 11. The completeness increases with $N_{3 R}$ from $18 \%$ to $29 \%$ for the M15 samples, and from $34 \%$ to $60 \%$ for the M14 samples. As for Figure 9, these fractions can be read off in Figure 11 from the ratio between the length of the blue sector of each bar and the total length of the bar.

Figure 12 shows the completeness as a function of the substructure mass for the M14 samples. Clearly, the most massive substructures are detected more easily, and more substructures are recognized in denser fields. The largest 3D substructure mass is $1.19 \times 10^{14} h^{-1} M_{\odot}$; this mass is larger than the maximum $M_{200}$ of the M14 sample, but it still is a factor of 2.2 smaller than the minimum total cluster mass $2.66 \times 10^{14} h^{-1} M_{\odot}$, which is defined as the sum of the mass of the dark matter and baryonic particles of the FoF halo, consistently with the computation of the mass of the 3D substructures. Figure 12 shows that we can obviously improve the completeness of our substructure sample by increasing the lower mass limit. The result is qualitatively similar to the results of the M15 samples, which we do not show here, although in this case, the trend has larger fluctuations and discontinuities due to the limited number of clusters and detected substructures.

To illustrate the origin of the relations plotted in Figure 12, we show two examples of the substructure mass function in Figure 13. The red histogram is the original mass function of 


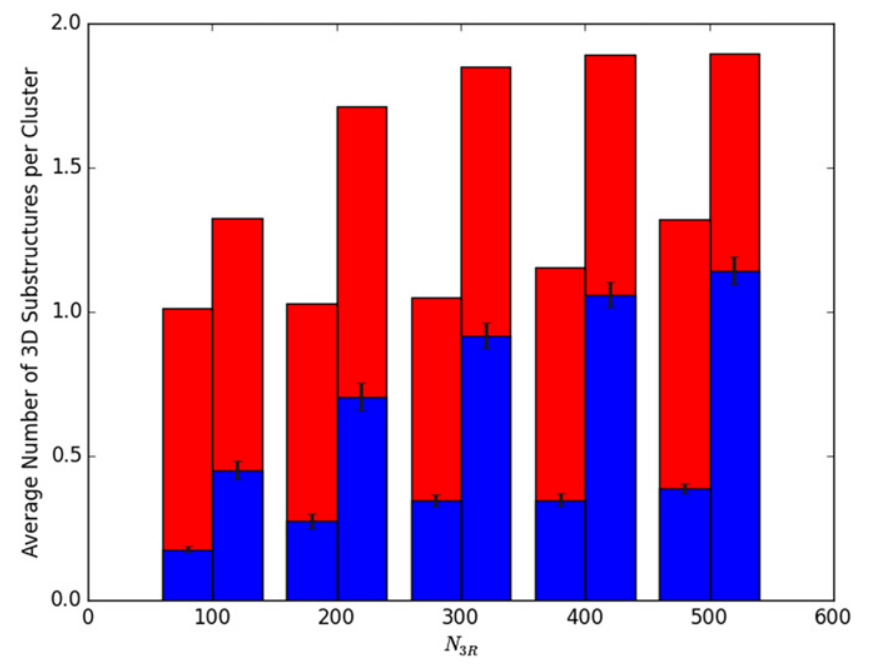

Figure 11. Completeness of the substructure catalogs. The left (right) bars are for the M15 (M14) samples. The blue sectors show the average number of 3D substructures properly detected in each cluster; the red sectors show the average number of missed 3D substructures in each cluster. The error bars show the $1 \sigma$ fluctuations from the ten random sampling realizations.

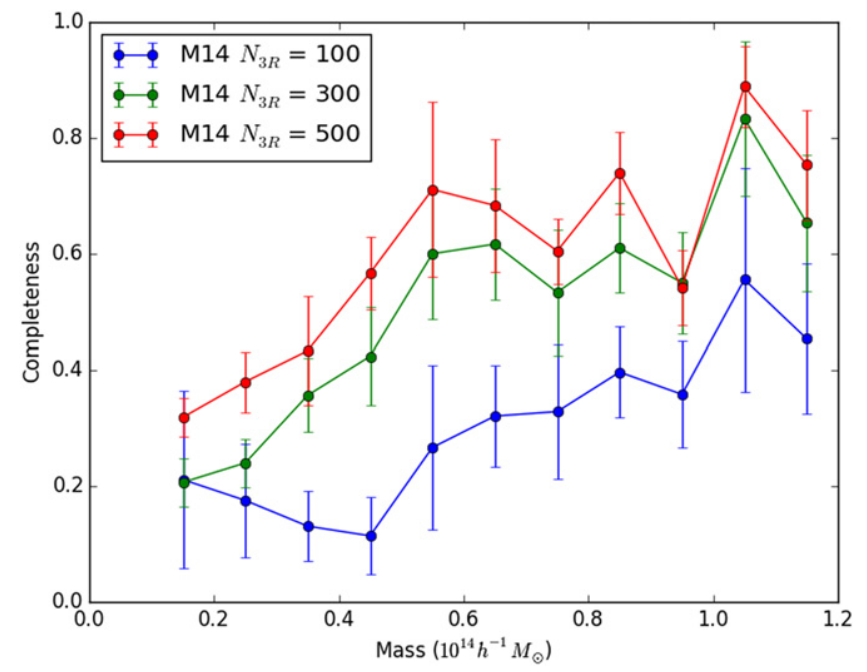

Figure 12. Completeness vs. 3D substructure mass in the M14 samples for three different $N_{3 R}$. The error bars show the $1 \sigma$ fluctuations from the ten random sampling realizations.

the 3D substructures in the 150-cluster M14 sample, whereas the blue histogram shows the estimated mass function: the original mass function is not monotonic and is severely underrepresented by the estimated mass function at the lowmass end.

This analysis of the dependence of the completeness on the minimum mass of the $3 \mathrm{D}$ substructure unfortunately cannot be repeated with the success rate. In fact, the substructure mass does not enter our algorithm for the identification of substructures from redshift data: changing the mass threshold would only change the number of $3 \mathrm{D}$ substructures, but it would leave the number of 2D substructures unaltered. Therefore, if we increase the mass threshold, the success rate simply decreases; if we decrease the mass threshold below $10^{13} h^{-1} M_{\odot}$, we start probing substructures with one or two bright galaxies at most, namely substructures that are virtually impossible to detect with current redshift surveys.

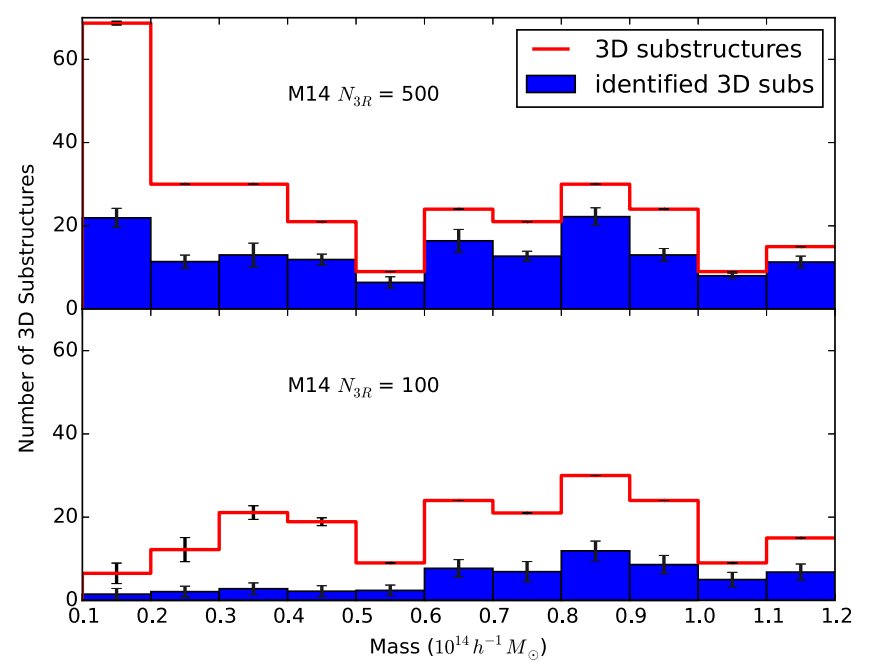

Figure 13. Substructure mass functions. The upper (lower) panel is from the M14 sample with $N_{3 R}=500$ (100). The open histograms show the real 3D substructure mass function, whereas the solid histograms show the mass function of the detected 3D substructures. The error bars show the $1 \sigma$ fluctuations from the 10 random sampling realizations.

\section{DISCUSSION}

\subsection{Possible Developments of the Caustic Technique}

In the previous sections we show that the caustic technique, when blindly applied to redshift surveys of clusters, provides encouraging values of the success rate and completeness. In principle, these results could be improved when a cluster is analyzed individually. In fact, the assumption that the two nodes, $x_{1}$ and $x_{2}$, and their corresponding thresholds alone are sufficient to separate the groups surrounding the cluster as well as the cluster substructures might be too simplistic in some clusters: their dynamical state can be complex enough that the deviation from the single isothermal sphere is relevant. In this case, looking for a single $\sigma$ plateau on the main branch might fail to capture the full information on the cluster dynamics contained in the binary tree.

For example, the velocity dispersion of the main group can substantially differ from the velocity dispersion of the substructures, and in turn different substructures can have different velocity dispersions. This situation can generate multiple $\sigma$ plateaus on the main branch and the determination of the proper thresholds is ambiguous. In these cases, the algorithm might not identify the main group and its substructures satisfactorily and might miss a substantial number of 3D substructures with velocity dispersions smaller than the velocity dispersions set by the $\sigma$ plateau.

Figure 14 illustrates this argument: it shows the distributions of the velocity dispersions of the 3D cores (green histograms), 3D substructures (red histograms), and the 2D substructures corresponding to 3D substructures (identified 3D substructures, blue histograms) for all of the combined M14 and M15 samples. The velocity dispersions of the 3D cores and substructures are computed with the full list of members provided by SUBFIND, whereas the velocity dispersion of the identified 3D substructures are computed from the list of members derived from the binary tree. In the M14 samples, the velocity dispersions of the cores and of the 3D substructures substantially overlap. Therefore the velocity dispersion corresponding to the single $\sigma$ plateau of the main branch of the 


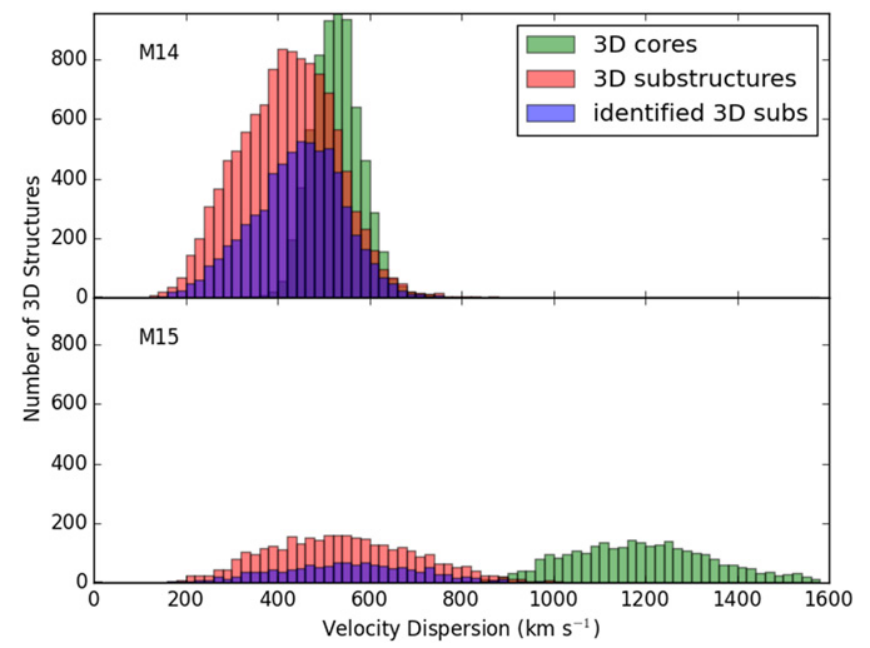

Figure 14. Distributions of the velocity dispersions of the 3D substructures (red histograms), 3D cluster cores (green histograms), and identified 3D substructures (blue histograms). The upper (lower) panel shows the M14 (M15) cluster samples.

binary tree can properly identify both the main group and the substructures. On the contrary, in the M15 samples, the distributions of the velocity dispersions of the cores and of the 3D substructures are almost completely separated and the $\sigma$ plateau that identifies the main branch is unlikely to also properly identify the substructures. Figure 14 thus suggests that this is the origin of most of the differences between the substructure identification results of the M14 and M15 samples that we describe in the previous sections.

In conclusion, the closer the velocity dispersions of the 3D substructures are to the velocity dispersion of their cores, namely to the $\sigma$ plateau, the more effectively our algorithm detects the 3D substructures. When applied to clusters individually, as can happen with real catalogs containing fewer clusters, if the shape of the velocity dispersion on the main branch versus the node number (Figure 2) is more complex than a single $\sigma$ plateau, the substructure detection can be improved by tuning the substructure threshold according to this shape. We plan to investigate this issue in detail in future work.

As a final note, we emphasize that our analysis is based on the assumption that a set of dark matter particles randomly sampled from an $\mathrm{N}$-body simulation is a fair representation of a real sample of galaxies. This assumption is expected to be valid at the high-mass end of the dark matter halo mass function, but it can become progressively incorrect at decreasing masses (Sawala et al. 2014). Therefore, luminosity and velocity segregations can partly differentiate the phase-space distributions of galaxies from those of an unbiased sample of dark matter particles. Associating methods, like the abundance matching technique (SHAM; Conroy et al. 2006; Vale \& Ostriker 2006), which assumes a monotonic relationship between observed galaxy luminosity and simulated halo mass, can be used to make tests on mock catalogs that resemble real data sets more closely. In future work we will explore this issue: we expect it to be particularly relevant in dealing with the different sizes of the M15 and M14 mock surveys (Table 1) and in addressing the dependence of the success rate on $N_{3 R}$ (Figure 9) in a more realistic context.

\subsection{Our Results in the Context of Previous Work}

Assessing the presence of substructures in clusters has been frequently investigated in the literature based on X-ray observations, weak gravitational lensing, or spectroscopic redshifts of galaxies. However, the issue has been addressed at very different levels.

X-ray analyses mainly focus on quantifying the surface brightness morphology, with the aim of either separating relaxed clusters from merging clusters (e.g., Parekh et al. 2015) or quantifying the systematic errors affecting cluster mass measurements (Nagai et al. 2007; Piffaretti \& Valdarnini 2008). The substructure identification is limited to the cluster central region within $r_{500}$, which is the typical largest distance where the ICM can be detected reliably (Piffaretti \& Valdarnini 2008), and to substructures that contain a quantity of hot gas large enough to produce a detectable X-ray emission. In addition, the identification of substructures is complicated by the fact that viscosity and magnetic fields can displace the hot gas from the dominant mass distribution, as indicated by the observations of numerous merging clusters (e.g., Markevitch et al. 2004; Mahdavi et al. 2007; Menanteau et al. 2012).

The mass distribution is expected to be directly detected, with studies based on weak gravitational lensing, whose signal is not affected by the complications of baryonic physics and the dynamical state of the cluster. However, unrelated large-scale structures along the line of sight (Hoekstra 2003; Hoekstra et al. 2011) and uncertainties on the lens parametrization (Clowe et al. 2004; Corless \& King 2007) can be sources of substantial systematic errors. For example, when weak lensing is applied to the identification of clusters in $\mathrm{N}$-body simulations, projection effects limit the fraction of identified clusters that correspond to real clusters to $20 \%$, whereas the completeness can be as large as $80 \%$ (White et al. 2002). Shirasaki (2015) proposes a method based on weak lensing to identify substructures in the outer regions of clusters, beyond 1 arcmin of the cluster center. The method is tested on $N$-body simulations where substructures are identified with SUBFIND. The fraction of real substructures that are identified with this algorithm is never larger than $40 \%$, approximately comparable to our results, whereas the contamination fraction is not mentioned.

The identification of substructures from the galaxy positions and redshifts has a long history, but the various algorithms have rarely been tested on realistic $N$-body simulations. Escalera \& Mazure (1992) test the wavelet transform method on 12 toy models where the particles of the main cluster are distributed according to a Hubble profile, with a Gaussian distribution of their velocities; only a single substructure, which is a rescaled model of the main cluster, is located away from the cluster core. The wavelet transform method yields a significance level of the identified substructure and Escalera \& Mazure (1992) show that for two of their significant substructures the fractions of particles that do not actually belong to the 3D substructures are $3 / 9$ and $0 / 7$.

Pinkney et al. (1996) investigate 31 different methods, including the DS and the KMM algorithms; only 5 out of these 31 methods use both galaxy redshifts and celestial coordinates. They build two-component merging clusters with $N$-body simulations and apply the methods at different epochs of the merging process and from different lines of sight. Therefore, the resulting mock fields of view contained a single substructure and no foreground and background large-scale 
structures or field particles. The main goal of their test is to check the efficiency of the substructure identification and the significance level of the detection. The DS method has the best performance, with a success rate of $66 \%$ at a $5 \%$ significant level, with a false detection rate of $\sim 10 \%$ in an isolated isothermal cluster. The success rate of the KMM algorithm is around $36 \%$, while the false detection rate rapidly increases with the number of particles within the mock catalog.

Systematic tests of the the DEDICA algorithm are performed by Ramella et al. (2007). They build artificial clusters with a single substructure away from the cluster center and use Poisson noise to simulate the background and foreground structures. The cluster and the substructure are spherically symmetric and have a King density profile. On these toy models, most of the time DEDICA can detect the substructure and recover $75 \%$ of its members.

Unlike the studies described above, we use mock redshift surveys that are extracted from a state-of-the-art $\mathrm{N}$-body simulation of a large cosmological volume, containing multiple substructures and foreground and background structures and filaments, thus providing very realistic mock fields of view. $\mathrm{N}$ body simulations similar to ours were adopted by weak lensing analyses (White et al. 2002; Shirasaki 2015). To match our 2D substructures with the 3D substructures, we apply a criterion based on the individual particles that are substructure members, a very strict but necessary criterion for assessing the efficiency of identifying the 3D substructures from 2D information. Our strict criterion applied to realistic mock fields of view is the most relevant difference between our analysis and previous work. Therefore, comparing our results with the results of other techniques by looking at their claimed performance alone can be misleading. In this realistic context, the ability of our method to identify roughly $30 \%-50 \%$ of the genuine substructures of the cluster, independently of its dynamical state and in the presence of coherent structures along the line of sight, is remarkable. It also is important to emphasize that our substructure identification method only uses the first step of the caustic technique, where the galaxies are arranged in a binary tree; this step does not assume any spherical symmetry, unlike the unused second part of the caustic technique where the redshift diagram and the caustic locations are determined. Therefore the substructure identification technique does not assume any specific geometry for the substructure.

False identifications and incompleteness appear to be unavoidable in any technique. In future work, we plan to investigate some possible improvements, mentioned in the previous subsection, that are expected to reduce these failures and enhance the performance of the caustic technique.

\section{CONCLUSIONS}

We test how efficiently the caustic technique can identify cluster substructures in mock redshift surveys of clusters extracted from $N$-body simulations. We consider two samples of 150 clusters each with $M_{200} \sim 10^{14} h^{-1} M_{\odot}$ (M14) and $M_{200} \sim 10^{15} h^{-1} M_{\odot}(\mathrm{M} 15)$. We consider mock redshift surveys with different numbers of particles $N_{3 R}$ within $3 R_{200}$, including $N_{3 R}=200$, which is the typical size of cluster redshift surveys like CIRS (Rines \& Diaferio 2006) and HeCS (Rines et al. 2013).

For the $N_{3 R}=200$ catalogs, among the 2D substructures identified by the caustic technique between 0.1 and $6 h^{-1} \mathrm{Mpc}$ from the cluster center, $17 \%$ and $21 \%$ correspond to the real $3 \mathrm{D}$ substructures with masses larger than $10^{13} h^{-1} M_{\odot}$ that are identified in three dimensions, for the M14 and M15 samples, respectively. These numbers represent a lower limit to the numbers of physically bound systems identified with the caustic technique because we also label as false detections real $3 \mathrm{D}$ substructures that are less massive than $10^{13} h^{-1} M_{\odot}$ or that belong to groups or clusters surrounding the cluster of interest. As for the completeness, the lists of 2D substructures contain $48 \%$ (M14) and 29\% (M15) of the 3D substructures that are more massive than $10^{13} h^{-1} M_{\odot}$ and with more than 10 particles in the FOV.

Our analysis shows that the completeness of the substructure catalog and the successful identification of substructures is a strong function of the substructure mass and the density of the survey. However, this latter parameter does not necessarily need to be as large as possible, because denser surveys have a larger probability of chance alignments and the association of interlopers. Quantifying these effects more systematically requires further investigation.

We show that the caustic method appears to be a promising technique for identifying substructures of galaxy clusters out to their outer regions from redshift surveys. When used for this purpose, the caustic technique does not require the assumption of spherical symmetry, and it is thus an ideal tool for analyzing complex systems. However, the method can certainly be improved: the caustic method arranges the galaxies in a binary tree based on a projected binding energy; the information on the dynamical state of the cluster contained in this binary tree is impressively rich and deserves further investigation to be fully exploited.

An efficient technique for investigating the substructure content of clusters is well-timed because data sets, including both redshift surveys of the large-scale structures, like SDSS (Ahn et al. 2014) and LAMOST (Zhao et al. 2012), and dedicated redshift surveys of clusters, like CIRS (Rines \& Diaferio 2006) and HeCS (Rines et al. 2013), are already available.

We sincerely thank Margaret Geller and Ken Rines for valuable suggestions and an anonymous referee whose detailed comments greatly helped us to illustrate our results more clearly. We acknowledge support from the grant Progetti di Ateneo/CSP - TO - Call2 - 2012_0011 "Marco Polo" of the University of Torino, the INFN grant InDark, the grant PRIN 2012 "Fisica Astroparticellare Teorica" of the Italian Ministry of University and Research, the Chinese Ministry of Science and Technology National Basic Science Program (Project 973) under grant No. 2012CB821804, the National Natural Science Foundation of China under grant No. 11403002, the Fundamental Research Funds for the Central Universities, and the Scientific Research Foundation of Beijing Normal University. M.B. also acknowledges the financial contribution by the PRIN INAF 2012 "The universe in the box: multiscale simulations of cosmic structure."

\section{REFERENCES}

Adami, C., Biviano, A., Durret, F., \& Mazure, A. 2005, A\&A, 443, 17 Aguerri, J. A. L., \& Sánchez-Janssen, R. 2010, A\&A, 521, A28 Ahn, C. P., Alexandroff, R., Allende Prieto, C., et al. 2014, ApJS, 211, 17 Andrade-Santos, F., Lima Neto, G. B., \& Laganá, T. F. 2012, ApJ, 746, 139 Ashman, K. M., Bird, C. M., \& Zepf, S. E. 1994, AJ, 108, 2348 Audit, E., Teyssier, R., \& Alimi, J.-M. 1998, A\&A, 333, 779 Baldi, M. 2012, MNRAS, 422, 1028 
Barmby, P., \& Huchra, J. P. 1998, AJ, 115, 6

Bird, C. 1994, ApJ, 422, 480

Bird, C. M., \& Beers, T. C. 1993, AJ, 105, 1596

Clowe, D., Bradač, M., Gonzalez, A. H., et al. 2006, ApJL, 648, L109

Clowe, D., De Lucia, G., \& King, L. 2004, MNRAS, 350, 1038

Colless, M., \& Dunn, A. M. 1996, ApJ, 458, 435

Conroy, C., Wechsler, R. H., \& Kravtsov, A. V. 2006, ApJ, 647, 201

Corless, V. L., \& King, L. J. 2007, MNRAS, 380, 149

Davis, M., Efstathiou, G., Frenk, C. S., \& White, S. D. M. 1985, ApJ, 292, 371

Diaferio, A. 1999, MNRAS, 309, 610

Diaferio, A. 2009, arXiv:0901.0868

Diaferio, A., \& Geller, M. J. 1997, ApJ, 481, 633

Diaferio, A., Kauffmann, G., Balogh, M. L., et al. 2001, MNRAS, 323, 999

Diemand, J., Moore, B., \& Stadel, J. 2004, MNRAS, 352, 535

Dressler, A., Oemler, A., Jr., Poggianti, B. M., et al. 2013, ApJ, 770, 62

Dressler, A., \& Shectman, S. A. 1988, AJ, 95, 985

Escalera, E., \& MacGillivray, H. T. 1995, A\&A, 298, 1

Escalera, E., \& Mazure, A. 1992, ApJ, 388, 23

Fasano, G., Poggianti, B. M., Bettoni, D., et al. 2015, MNRAS, 449, 3927

Feretti, L., Giovannini, G., Govoni, F., \& Murgia, M. 2012, A\&ARv, 20, 54

Flin, P., \& Krywult, J. 2006, A\&A, 450, 9

Gambera, M., Pagliaro, A., Antonuccio-Delogu, V., \& Becciani, U. 1997, ApJ, 488,136

Geller, M. J., \& Beers, T. C. 1982, PASP, 94, 421

Geller, M. J., Diaferio, A., Rines, K. J., \& Serra, A. L. 2013, ApJ, 764, 58

Gill, S. P. D., Knebe, A., \& Gibson, B. K. 2005, MNRAS, 356, 1327

Gill, S. P. D., Knebe, A., Gibson, B. K., \& Dopita, M. A. 2004, MNRAS, 351, 410

Girardi, M., Escalera, E., Fadda, D., et al. 1997, ApJ, 482, 41

Girardi, M., Fadda, D., Giuricin, G., et al. 1996, ApJ, 457, 61

Girardi, M., Mercurio, A., Balestra, I., et al. 2015, A\&A, 579, A4

Hoekstra, H. 2003, MNRAS, 339, 1155

Hoekstra, H., Franx, M., \& Kuijken, K. 2000, ApJ, 532, 88

Hoekstra, H., Hartlap, J., Hilbert, S., \& van Uitert, E. 2011, MNRAS, 412, 2095

Huchra, J. P., \& Geller, M. J. 1982, ApJ, 257, 423

Jeltema, T. E., Canizares, C. R., Bautz, M. W., \& Buote, D. A. 2005, ApJ, 624, 606

Kaiser, N. 1987, MNRAS, 227,

Kauffmann, G., \& White, S. D. M. 1993, MNRAS, 261, 921

Knebe, A., \& Müller, V. 2000, A\&A, 354, 761

Kneib, J.-P., Ellis, R. S., Smail, I., Couch, W. J., \& Sharples, R. M. 1996, ApJ, 471,643

Kolokotronis, V., Basilakos, S., Plionis, M., \& Georgantopoulos, I. 2001, MNRAS, 320, 49

Komatsu, E., Matsuo, H., Kitayama, T., et al. 2001, PASJ, 53, 57

Korngut, P. M., Dicker, S. R., Reese, E. D., et al. 2011, ApJ, 734, 10

Kriessler, J. R., \& Beers, T. C. 1997, AJ, 113, 80

Mahdavi, A., Hoekstra, H., Babul, A., Balam, D. D., \& Capak, P. L. 2007, ApJ, 668,806

Mao, S., Jing, Y., Ostriker, J. P., \& Weller, J. 2004, ApJL, 604, L5
Mao, S., \& Schneider, P. 1998, MNRAS, 295, 587

Markevitch, M., Gonzalez, A. H., Clowe, D., et al. 2004, ApJ, 606, 819

McCleary, J., dell'Antonio, I., \& Huwe, P. 2015, ApJ, 805, 40

Menanteau, F., Hughes, J. P., Sifón, C., et al. 2012, ApJ, 748, 7

Mohr, J. J., Evrard, A. E., Fabricant, D. G., \& Geller, M. J. 1995, ApJ, 447, 8

Nagai, D., Vikhlinin, A., \& Kravtsov, A. V. 2007, ApJ, 655, 98

Oguri, M., Schrabback, T., Jullo, E., et al. 2013, MNRAS, 429, 482

Okabe, N., Takada, M., Umetsu, K., Futamase, T., \& Smith, G. P. 2010, PASJ, 62,811

Pagliaro, A., Antonuccio-Delogu, V., Becciani, U., \& Gambera, M. 1999, MNRAS, 310, 835

Parekh, V., van der Heyden, K., Ferrari, C., Angus, G., \& Holwerda, B. 2014, arXiv: 1411.6525

Parekh, V., van der Heyden, K., Ferrari, C., Angus, G., \& Holwerda, B. 2015, A\&A, 575, A127

Pastor Mira, E., Hilbert, S., Hartlap, J., \& Schneider, P. 2011, A\&A, 531, A169

Piffaretti, R., \& Valdarnini, R. 2008, A\&A, 491, 71

Pinkney, J., Roettiger, K., Burns, J. O., \& Bird, C. M. 1996, ApJS, 104, 1

Pisani, A. 1993, MNRAS, 265, 706

Pisani, A. 1996, MNRAS, 278, 697

Ramella, M., Biviano, A., Pisani, A., et al. 2007, A\&A, 470, 39

Regös, E., \& Geller, M. J. 1989, AJ, 98, 755

Richstone, D., Loeb, A., \& Turner, E. L. 1992, ApJ, 393, 477

Rines, K., \& Diaferio, A. 2006, AJ, 132, 1275

Rines, K., Diaferio, A., \& Natarajan, P. 2008, ApJL, 679, L1

Rines, K., Geller, M. J., Diaferio, A., \& Kurtz, M. J. 2013, ApJ, 767, 15

Salvador-Solé, E., González-Casado, G., \& Solanes, J. M. 1993, ApJ, 410, 1

Sawala, T., Frenk, C. S., Fattahi, A., et al. 2014, arXiv:1404.3724

Schuecker, P., Böhringer, H., Reiprich, T. H., \& Feretti, L. 2001, A\&A, 378, 408

Serna, A., \& Gerbal, D. 1996, A\&A, 309, 65

Serra, A. L., \& Diaferio, A. 2013, ApJ, 768, 116

Serra, A. L., Diaferio, A., Murante, G., \& Borgani, S. 2011, MNRAS, 412, 800

Shane, C. D., \& Wirtanen, C. A. 1954, AJ, 59, 285

Shirasaki, M. 2015, ApJ, 799, 188

Slezak, E., Bijaoui, A., \& Mars, G. 1990, A\&A, 227, 301

Solanes, J. M., Salvador-Solé, E., \& González-Casado, G. 1999, A\&A, 343, 733

Springel, V., White, S. D. M., Tormen, G., \& Kauffmann, G. 2001, MNRAS, 328,726

Thomas, P. A., Colberg, J. M., Couchman, H. M. P., et al. 1998, MNRAS, 296, 1061

Vale, A., \& Ostriker, J. P. 2006, MNRAS, 371, 1173

van Haarlem, M., \& van de Weygaert, R. 1993, ApJ, 418, 544

West, M. J., \& Bothun, G. D. 1990, ApJ, 350, 36

West, M. J., Oemler, A., Jr., \& Dekel, A. 1988, ApJ, 327, 1

White, M., van Waerbeke, L., \& Mackey, J. 2002, ApJ, 575, 640

Zhang, Y.-Y., Reiprich, T. H., Finoguenov, A., Hudson, D. S., \& Sarazin, C. L. 2009, ApJ, 699, 1178

Zhao, G., Zhao, Y.-H., Chu, Y.-Q., Jing, Y.-P., \& Deng, L.-C. 2012, RAA, 12,723 